\title{
A Proficient Technique for Extraction the High Average-Utility Itemsets with Enhanced Bounds From Transactional Database
}

\author{
L. Chandana, P. Radhika
}

\begin{abstract}
HUIM has turned into a well known knowledge extraction, as it can uncover designs that have a highutility, conversely to continuous example extraction, which spotlights on finding incessant examples. High average-utility itemset extraction (HAUIM) is different with HUIM gives an elective quantify, called the average utility, to choose designs by considering their utilities and lengths. In the most recent decades, a few calculations have been created to mine HAUIs. However majorly it takes lot of memory and time, since they for the most part use the average-utility upper-bound model to miscalculate the average utilities of itemsets. To enhance HAUIM here proposes four average utility upper bounds, in view of structure database portrayal, and three proficient prune techniques. Furthermore, a novel conventional system for looking at average-utility upper-bounds is displayed. In view of these theoretical outcomes, a proficient calculation named dHAUIM is presented for extraction the total arrangement of HAUIs. dHAUIM speaks to the inquiry space and rapidly process upper-bounds utilizing a novel IDUL structure. Broad investigations demonstrate that dHAUIM beats three algorithms for extraction HAUIs as far as runtime on both reality and artificial databases.
\end{abstract}

Keywords : Pattern extraction, utility extraction, high average-utility

\section{INTRODUCTION}

The primary reason for data extraction methods is to uncover significant, intriguing and possibly valuable data in enormous databases [1], [2], [11]. "FIM just consider event frequencies of itemsets, and does not consider other variables that can assess the significance of itemsets, for example, buy amounts, unit profits of items, and for the most part the intriguing quality or loads of items". Accordingly, the data separated by customary FIM calculations is lacking for some applications.

To uncover increasingly valuable and significant data from transactional database, the errand of HUIM was proposed by Yaoet al. It thinks about both buy amounts and unit profits of items, to locate the arrangement of HUIs. In HUIM, thing amounts in a database are known as the inward utilities of items, and the thing unit profits are known as the outer utilities.

Revised Manuscript Received on July 22, 2019.

L. Chandana, P. Radhika

M. Tech, Assistant Professor

Department of Computer Science and Engineering,

VNR Vignana Jyothi Institute of Engineering \& Technology,
EHAUPM is "Tighter Upper Bounds Trade weighted usage itemsets (HTWUIs) as potential HUIs. A few calculations [3], [7] proposed to mine HUIs utilizing the TWU model and extra upgrades have been proposed to accelerate their presentation". Despite the fact that HUIM can uncover progressively valuable data contrasted with customary FIM, it experiences a significant issue. It is that the length of each itemset isn't viewed as when estimating its utility. This is uncalled for as the utilities of itemsets will in general be more noteworthy for itemsets containing more items. To address this issue and give an all the more reasonable estimation of the utilities of itemsets for genuine applications, HAUIM [15] was proposed.

The objective of HAUIM is to locate the arrangement of all high. Honget al. [15]" first structured a two-stage TPAU calculation to mine HAUIs utilizing an Apriori like methodology. The auub model was intended to guarantee the culmination and accuracy of the calculation for extraction HAUIs. To accelerate HAUIM, a projection-based PAI calculation, a tree-based high average-utility example HAUP-tree calculation [22], and a HAUI-tree calculation were intended to productively mine HAUIs, in light of the TPAU calculation. The HAUI-Miner calculation was then created to further upgrade the extraction execution utilizing a planned AU list structure. This calculation is at present the state-of-the-art HAUIM algorithm". Nonetheless, HAUI-Miner experiences the issue of playing out an exorbitant join activity various occasions for extraction HAUIs since it receives the auub model to overestimate the utility of itemsets.

1) Two more tightly "upper-bounds are proposed to significantly lessen the search space for extraction HAUIs. The lub model is first intended to consider the average-utilities of itemsets and their staying greatest utilities in exchanges. The second rtub model is additional intended to disregard immaterial itemsets in exchanges, which can be utilized to further diminish the search space for extraction HAUIs".

2) An "adjusted average-utility list structure is created to decrease the quantity of database outputs and store the required data for extraction HAUIs. Three pruning methodologies are likewise 
created to improve the exhibition of HAUI extraction utilizing the two planned models and MAU-list organization".

3) General trials are "directed on both genuine world and synthetic datasets to demonstrate that the proposed calculation altogether outflanks the state-of-the-art HAUI-Miner calculation regarding runtime, memory utilization, number of join tasks and adaptability".

\section{LITERATURE SURVEY}

\section{[1] Unil Yun and John J. Leggett}

Analysts proposed the WFIM calculation to cast back the significance of itemset. It for the most part center on the loads of the itemsets fulfilling the downwards conclusion property. Till now all the weighted itemset extraction is relies on the Apriori calculation. The scope of the loads and the base weight confinement are characterized in which everything is relegated with the abnormal loads in the scope of the weight. WFIM produce concise data identified with the weighted regular itemset in huge database, by modifying least weight and weight territory.

\section{[2] U. Yin}

Extraction of frequent pattern of the calculations uses bolster requirements with combinational hunt space yet only it isn't sufficient. The incessant examples which got in the wake of extraction result into the examples of feeble closeness. Regardless of expanding the base help related examples were not recognized totally. To recognize the related examples intriguing examples are proposed. Another procedure named weight intrigue example extraction is exhibited which gives weight certain for creating the connected examples. WIP gives low computational expense, without utilizing the upper bound it utilizes the novel h-certain. The resultant yield of this extraction furnishes with lesser patter however with high connection and adaptable. Contingent on the application prerequisite the h-sure, weight backing and edge esteems are decreased.

\section{[3] Chowdhury Farhan Ahmed, Syed Khairuzzaman} tanbeer

In the exploration zone of the data extraction, HUP is a noteworthy critical issue as it takes nonexistent recurrence and each estimation of profit various items from exchange. To diminish trivial figurings the least edge esteems are set and the database is refreshed relying on the expansion and common extraction of data gives past data structure. An epic tree structure was proposed with three tree structure to play out the extraction. The principal tree sort out items in the lexicographical request which catches the data without extra remodel activities. The subsequent tree structure gives compacted size of items relying on the recurrence of it in exchange. The third tree structure utilizes exchange weight tree which can trim down the extraction time of the items. This tree structure gives adroit and adaptable results however this examination.

\section{[4] Tzung-Pei Hong, Cho-Han Lee}

Extraction distinguishes the itemsets whose occurrence is standard in the database keeping aside the various components like profit and cost. In increment to inclusion of expense, profit and other processable client necessities an utility pattern was proposed. Here delivers the averagely utilized of the items and don't permit to have down ward conclusion property. The most extreme summing up is taken as the constraint which is utilized for calculations of items later the quantity of items are cut off by applying some edge esteems which is a two stage process.

\section{[5] Mengchi Liu , Junfeng Qu}

The items which are for the most part utilized are perceived database relying on its factors. To distinguish such most utilized items a few algorithms have been proposed which gives the result by creating the quantity of applicant sets and portraying the items as constant items, by evaluating extremely that the thing is utilized increasingly number of times. In fact the candidates set which are delivered are not exact taking about off base result. So to conquer this miner-HUI algorithm was proposed which gains the data identified with items which are utilized number of times and pruning data in regards to the hunt space. The results of the algorithm were contrasted and other following in less utilization of memory and run time.

\section{IMPLEMENTATION METHODOLOGY}

Novel Pruning Strategies based on the Proposed UBs

Here $a u b 1$, and $i a u b$ are individually bit by bit more tightly UBs on $a u$, and $H A U S \subseteq H I A U B \subseteq H A U B \subseteq$ $H A U B 1$, "each UB has its very own pruning capacity or impact. For any $\mathrm{UB} u b$, we state that pruning condition $(P)$ for a non-void itemset $P$ holds if $u b(P)<m u$ (or $P$ is low-ub). For quickness, we signify the set of $P$ and all itemset augmentations of $P$ as $\operatorname{branch}(P)$ ". Furthermore, "the documentation $[P] \stackrel{\text { def }}{=}\{P x, \ldots, \ldots, \ldots\}$ will signify the identicalness class comprising of all thing augmentations of $P$ (itemsets having a similar prefix $P$ ). In light of these ideas, the inquiry space of HAUIM can be seen as a prefix-tree, where every hub speaks to an itemset, with the end goal that the root is the unfilled set and every offspring of a hub is single thing expansion of that itemset".

Depth Pruning strategy w.r.t. Forward augmentations dependent on "iaub and lau For any non-void itemset $P$ if $P C i(P)$ or $P C l a u b(P)$ hold the entire $\operatorname{branch}(P)$ of the identified space can be prune. Since $i a u b$ and $l a u b$ are unique, both of these UBs ought to be utilized to dispose of without prospect competitor parts of the prefix-tree".

Width Pruning strategy w.r.t. bi-directional augmentations on anticipated databases based onaub). For the second UBaub, "which is 
bigger than iaub, if $P C a u b(R y)$ holds for a non-void itemset $R$ and thing expansion $R y$ in $[R]$, the itemset $R y$ can be expelled from the set $[R]$, for example the entire $\operatorname{branch}(R y)$ is promptly pruned, yet additionally all branches $\operatorname{branch}(R x y)$ and $\operatorname{branch}(R y z)$ are wiped out from the search tree" (where $R x y$ and $R y z$ are individually the regressive and forward expansions of Ry).

Strong Width Pruning strategy On the underlying QDB $\mathcal{D}$ based onaub1. For the "aub1 UB, which is the biggest among the four new UBs, on the off chance that PCaub1 $(P)$ holds, then $P C a u b 1(C)$ additionally holds for all augmentations $C$ of $P$ '. In particular, for everything $a j$ of $\mathcal{A}$, if $P C a u b 1(a j)$ holds, for example $a u b 1(a j)<m u$, and we can expel $a j$ from the database $\mathcal{D}$ or erase the $j t h$ segment (as per $a j$ ) of the incorporated grid $\mathcal{Q}$.

Note that, in spite of the fact that $a u b 1$ and $a u u b$ have the equivalent SWP pruning capacity, aub1 is more tightly than $a u u b$ by Theorem 1.e. In this way, as far as worth and search space pruning capacity, $a u b 1$ is said to be totally superior to auub.

Clearly, "SWP and WP are separately more grounded than WP and DP. Albeit both $a u b 1$ and $a u b$ have the WP capacity, $a u b$ is just utilized on anticipated databases for non-void prefixes while $a u b 1$ can be also connected on the biggest beginning QDB $\mathcal{D}$ " (as per the vacant prefix).

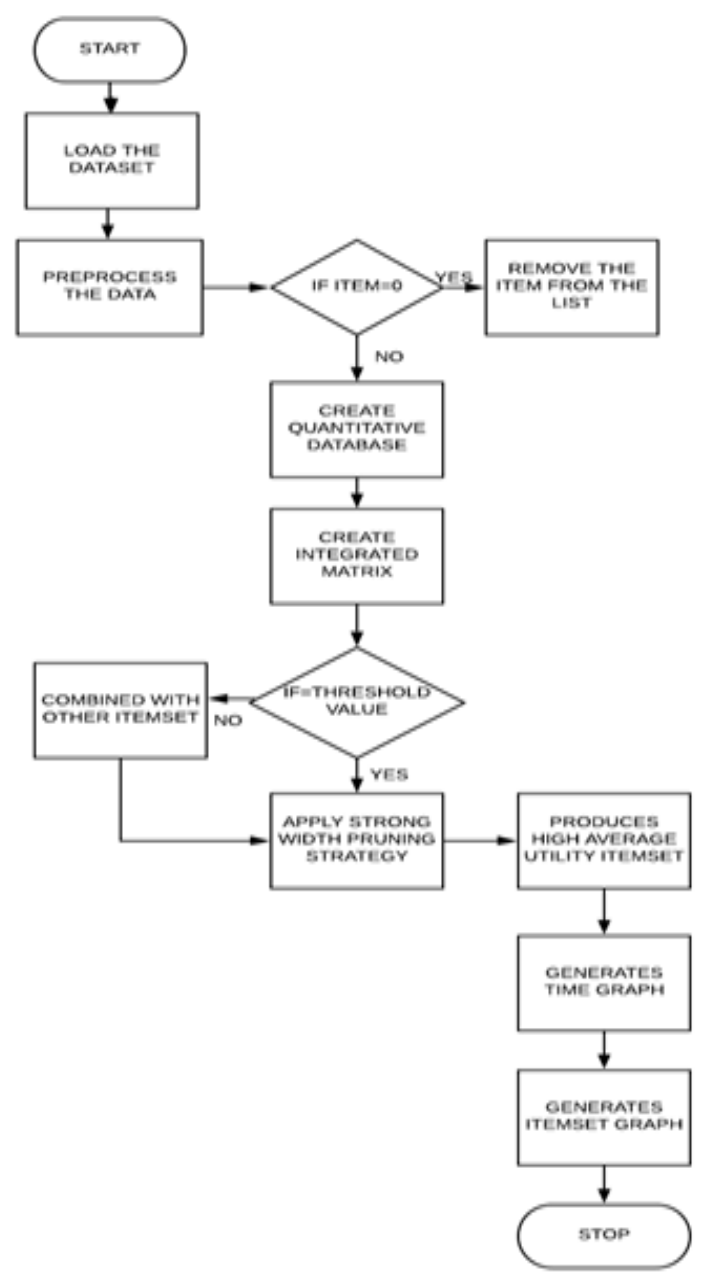

Fig: Implementation System

Primers in "high average utility patterns Let $\mathrm{I}=\{\mathrm{i} 1$, $\mathrm{i} 2, \mathrm{i} 3 \ldots \ldots \ldots . . . \mathrm{im}\}$ be a set of items. Let $\mathrm{D}=\{\mathrm{T} 1, \mathrm{~T} 2$, T3......Tn $\}$ be where $\mathrm{Ti}$ speaks to exchanges. Let $\mathrm{P}=\{\mathrm{P}(\mathrm{i} 1), \mathrm{P}(\mathrm{i} 2)$..........p(im) $\}$ be a unit of items I. Give $\mathrm{X}$ a chance to be an itemset contain items im and let $\mathrm{k}$ be the length of itemset. The length of itemset is the quantity of items in X'. Let $\mu$ be a base average-utility limit. Primer definitions for figuring utility estimation of itemsets are as per the following [1-5]:

Description 1: Utility of an item is amount of items in an trade the database.

Description 2: Outside utility of a thing is a part profit of a item.

Description 3: Utility capacity $\mathrm{f}$ is the result of inner and outside utility

Description 4: Utility of thing in trade $\mathrm{T}$ is the utility capacity of the thing in that particular exchange.

\section{dHAUIM algorithm}

In view of the novel $a u b 1$, iaub and laub UBs and the recursive recipes of Proposition 3, this area shows a proficient algorithm, named dHAUIM, "for extraction the set of all HAUIs, HAUS $\stackrel{\text { def }}{=}\{(C, a u(C))$ | $a u(C) \geqslant m u\}$. The proposition of this algorithm responds to the third research question $(Q 3)$. The pseudocode of the algorithm is appeared in roar. During the extraction expertness, HAU candidate itemsets are put away in a prefix-tree using a novel structure named IDUL. This structure contains the data of a hub $C$ of the structure $(C, \quad(C),(C))$ ". Review that the documentation $[P]$ signifies the set of thing expansions of a parent hub $P$.

\section{Algorithm1: $H A U S$ dHAUIM(D, $m u)$}

Input: a QDB $\mathcal{D}$, minimum $\mathrm{AU}$ threshold $m u$.

Output: "set of high-average utility itemsets HAUS".

1. Generate integrate matrix $2 n x m$.

2. Scan $Q n x m$ once to calculate the vectors $(\phi)$ and (aj) for each aj $\in \mathcal{A}$;

3. $[\phi]=\{(a j,(a j),(a j)) \mid a j \in \mathcal{A}$ and $a u b \overline{1(a j)} \geqslant$ $m u\}$;

//strong width pruning

4. $H A U S=\phi$;

5. HAU-Search $([\phi], H A U S)$;

6. return HAUS;

\section{Algorithm2: HAU-Search([P], HAUS)}

Input: "set $[P]$ of all item-extensions of $P$, the set HAUS".

Oucomet: the updated HAUS set.

1. $\operatorname{if}([P] \neq \phi)$ then \{

2. for each $(C i, d(C i), \mathcal{V}(C i))$ in $[P]$ do \{

3. if $(i a u \overline{b(} C i) \geqslant m u$ or $\operatorname{laub}(C i) \geqslant m u)$ then \{ //depth pruning

4. if $(\mathrm{au}(\mathrm{Ci})) \geqslant \mathrm{mu}))$ then

5. HAUS.Add (Ci, au(Ci)); 
6. $\operatorname{if}(|[\mathrm{P}]|>1)$ then \{

7. $[\mathrm{Ci}]=\phi$;

8. for $\operatorname{each}(C j, d(C j), \mathcal{V}(C j))$ in $[P]$, with $j>i \operatorname{do}\{$

9. $E=C i \cdot C j ;(E)=d(C j) \backslash d(C i)$;

10. Calculate V (E);

11. if $(a u b(E) \geqslant m u)$ then//width pruning

12. $[C i]=[C i] \cup\{(E, d(E), \mathcal{V}(E)\}$;

13. $\}$

14. HAU-Search([Ci], HAUS);

15. \} \}

17. \} \}

19. return;
Experimental Analysis

In the analysis tried different datasets particularly online retail, and a "few synthetic datasets are utilized, where online retail is accessible at the UCI store. The datasets contains genuine exchanges with synthetic utility qualities while online retail contains genuine utility qualities and exchanges happening somewhere in the range of 2014 and 2015 for a UK-based and enrolled non-store online retail".

Table1: Dataset Details

\begin{tabular}{|c|c|c|c|c|c|c|}
\hline 1 & Invoive No & Stock Code & Product & Quantity & Unit Price & Customer Id Country \\
\hline 2 & 536367 & 84879 & ASSORTED COLOUR BIRD ORNAMENT & 32 & 169 & 13047 UNITED KINGDOM \\
\hline 3 & 536374 & 21258 & VICTORIAN SEWING BOX LARGE & 32 & 1095 & 15100 UNITED KINGDOM \\
\hline 4 & 536376 & 22114 & OT WATER BOTTLE TEA AND SYMPATHY & 48 & 345 & 15291 UNITED KINGDOM \\
\hline 5 & 536378 & 84991 & 60 TEATIME FAIRY CAKE CASES & 24 & 125 & 14690 UNITED KINGDOM \\
\hline 6 & 536378 & 21977 & PACK OF 60 PINK PAISLEY CAKE CASES & 24 & 55 & 14688 UNITED KINGDOM \\
\hline 7 & 536378 & $85071 B$ & RED CHARLIELOLA PERSONAL DOOR SIGN & 96 & 38 & 14676 UNITED KINGDOM \\
\hline 8 & 536378 & $851838 \mathrm{~B}$ & CHARLIE LOLA WASTE PAPER BIN FLORA & 48 & 125 & 14888 UNITED KINGDOM \\
\hline 9 & 536378 & 21094 & SET 6 RED SPOTTY PAPER PLATES & 12 & 85 & 14666 UNITED KINGDOM \\
\hline 10 & 536371 & 22086 & PAPER CHAIN KIT50S CHRISTMAS & 80 & 255 & 13748 UNITED KINGDOM \\
\hline 11 & 536376 & 21733 & RED HANGING HEART T-LIGHT HOLDER & 64 & 255 & 15291 UNITED KINGDOM \\
\hline
\end{tabular}

Runtime: "The runtime of the proposed dHAUIM algorithm is contrasted and those of EHAUPM, D-FHAUM, MHAI and HAUI-Miner for different characteristics esteems on both reality and synthetic datasets. Results find that the runtimes of the algorithms decline as $\mathrm{mu}$ is expanded. The reason is that the quantities of HAUIs (\#HAUIs) discovered and join activities performed normally decline as $\mathrm{mu}$ is expanded".

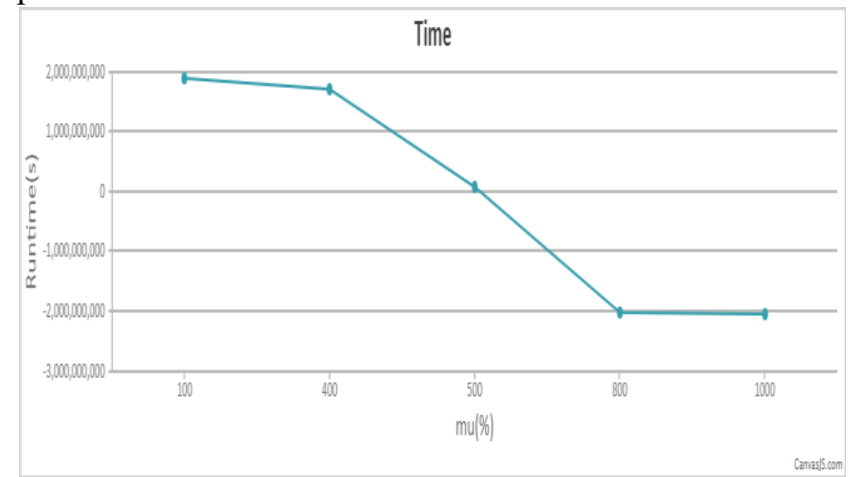

Number of join activities: "The quantity of join tasks performed by the algorithms was additionally recorded for all datasets and different properties esteems. Results the quantity of join tasks of dHAUIM is considerably less than that of the past algorithms MHAI, D-FHAUM, EHAUPM and HAUI-Miner. Along these lines, the search space of dHAUIM can be de-wrinkled drastically. Thus, dHAUIM is likewise a lot quicker than the other algorithms. Here is expanded from 0.42 to $0.9 \%$, the quantity of join activities performed by dHAUIM is 69.4to 98.8\%less than the past state-of-the-art algorithms".

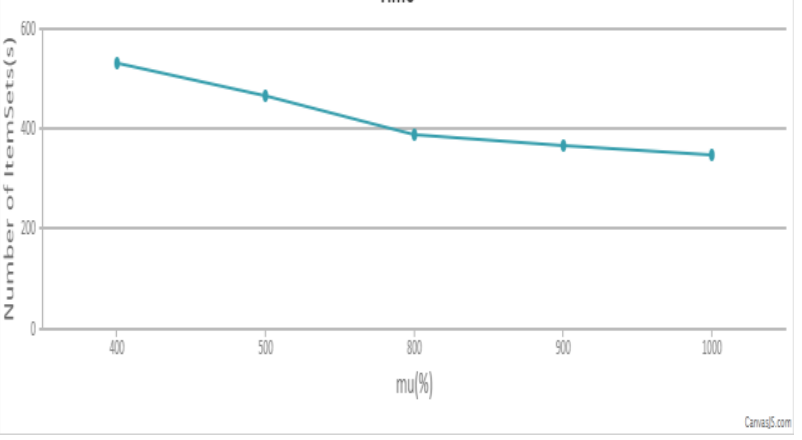

\section{CONCLUSION}

In view of registering utility qualities utilizing a structure in quantitative databases, this paper has presented four UBs, called $a u b 1$, $a u b$, iaub and $l a u b$, a conventional system to assess UBs regarding their pruning's, and three pruning methodologies to take out unpromising candidates early. "Another IDUL tree structure was likewise created to rapidly ascertain the average utility and UBs of itemsets utilizing a recursive procedure. An epic algorithm named dHAUIM has been further displayed to productively mine high average-utility itemsets. A broad trial assessment was done. Results have demonstrated that dHAUIM outflanks four state-of-the-art HAUI extraction algorithms as far as execution time and number of join tasks on both reality and synthetic databases". 


\section{REFERENCES}

[1] Unil Yun, John J, Leggett, "WFIM: Weighted Frequent Itemset Extraction with a weight range and a minimum weight" in SIAM International Conference on Data Extraction, 2005

[2] Unil Yun, "Efficient extraction of weighted interesting patterns with a strong weight and/or support affinity," in information science, volume 177, issue 17, 1 September 2007

[3] Chowdhury Farhan Ahmed, Syed Khairuzzaman Tanbeer , Byeong-Soo Jeong, Young-Koo Lee, "Efficient Tree Structures for Highutility Pattern Extraction in Incremental

Databases," in IEEE Transactions on Knowledge and Data Engineering ,volume 21, No (12), January 2010

[4] Tzung-PeiHong, Cho-HanLee, Shyue-LiangWang, "Effective utility extraction with the measure of average utility " in Expert Systems with Applications Volume 38, Issue 7, July 2011.

[5] Mengchi Liu, Junfeng Qu, "Extraction Highutility Itemsets without Candidate Generation", in ACM international conference on Information and knowledge management, October 2012

[6] Guo-Cheng Lan, Tzung-Pei Hong, Hong Yu Lee, Shyue-Liang Wang, Chun-Wei Tsai, "Enhancing the Efficiency in Extraction Weighted Frequent Itemsets", in Systems Man and Cybernetics (SMC) IEEE International Conference, 2013.

[7] R. Agrawal, T. Imielinksi and A. Swami, "Extraction Association Rules between Sets of Items in Large Database," in The ACM SIGMOD International Conference on Management of Data, 1993.

[8] V. Torres, K. Chiu and M. Vasudeva, "WFIM: weighted frequent itemset extraction with a weight range and a minimum weight," in The 2005 SIAM International Conference on Data Extraction, 2005.

[9] U. Yun, "Efficient extraction of weighted interesting patterns 